\title{
Primary Surgery Compared to Post Chemotherapy Surgery for Patients with Locally Advanced Breast Cancer
}

\author{
Ibrahim Altedlawi AlBalawi \\ Department of Surgery, Faculty of Medicine, University of Tabuk, Tabuk, Saudi Arabia \\ Email: drbalawi@yahoo.com
}

How to cite this paper: AlBalawi, I.A. (2018) Primary Surgery Compared to Post Chemotherapy Surgery for Patients with Locally Advanced Breast Cancer. Journal of Cancer Therapy, 9, 780-792. https://doi.org/10.4236/jct.2018.910064

Received: September 7, 2018

Accepted: October 5, 2018

Published: October 8, 2018

Copyright $\odot 2018$ by author and Scientific Research Publishing Inc. This work is licensed under the Creative Commons Attribution International License (CC BY 4.0).

http://creativecommons.org/licenses/by/4.0/ (c) (i) Open Access

\begin{abstract}
Purpose: To compare primary surgery and surgery after neoadjuvant chemotherapy (NCT) in locally advanced breast cancer (LABC) patients. Methods: Between January 2011 \& December 2015, 112 patients with LABC were treated at KAAH \& OC-Jeddah-KSA, Of whom 42 were treated by NCT followed by surgery either mastectomy or conservative surgery, then adjuvant chemotherapy and radiotherapy. The rest patients (70) were treated by primary surgery (mastectomy or conservative resection) and followed by adjuvant chemotherapy and radiotherapy. All patients received adjuvant antiestrogen. Patients were followed for a median duration of 33 months. Disease-free survival (DFS) and overall survival (OAS) were studied for all patients, compared between both groups and related to the extent of surgery and menopausal status. Results: median age was 46.5 years for all patients; 48 years, and 46 years for NCT and primary surgery groups respectively. Median DFS was 15 months for all patients, $16 \& 15$ months for NCT and primary surgery groups. Median OAS was 24 months for all patients, $22 \& 24$ months for NCT and primary surgery groups. Difference in DFS \& OAS were highly significant in favour of postmenopausal patients $(p=0.05$ for DFS \& $p=0.03$ for OAS) while in primary surgery group the differences between pre and postmenopausal patients in DFS \& OAS were statistically insignificant ( $\mathrm{p}=$ 0.4). NCT followed by surgery group patients showed significant improvement in DFS \& OAS in patients performed conservative surgery while in primary surgery group the difference was insignificant. Freedom of disease was seen in $28.6 \%$ in the NCT group and $37.1 \%$ for the primary surgery group. Conclusion: Surgery post-neoadjuvant chemotherapy neither prolongs DFS nor OAS in comparison with primary surgery followed by adjuvant chemotherapy. Postmenopausal patients felt much better than premenopausal patients regarding DFS \& OAS.
\end{abstract}




\section{Keywords}

Adjuvant, Breast, Cancer, Neoadjuvant, Surgery

\section{Introduction}

Locally advanced breast carcinoma (LABC) comprises a heterogeneous group of tumors ranging from relatively large primary tumors (stage T4) to small breast tumors presenting with extensive nodal metastases (involvement of ipsilateral, infraclavicular, supraclavicular, or internal mammary nodes). It remains a clinical challenge as the majority of patients with this diagnosis develop distant metastases despite appropriate therapy [1] [2]; inflammatory carcinomas also included in locally advanced breast carcinoma [3]. It is defined by 1992 American Joint Committee (AJC) staging criteria for stage IIIa and IIIb disease [4]. Despite the awareness of physician and public of the importance of screening and early detection, $10 \%-20 \%$ of women with breast cancer have the locally advanced disease at diagnosis in industrialized countries (14\% in the United States) while in developing countries it might constitute up to $50 \%$ of incident cases [5]. In populations that receive regular screening mammography, the percentage of patients with the locally advanced disease is less than 5\% [6]. The treatment for patients with locally advanced breast cancer is typically a combination of systemic chemotherapy, surgery, and radiotherapy. There is a consensus that all patients with the technically resectable disease should have radical mastectomy [4]. Earlier results of radical mastectomy alone were associated with a 53\% local failure rate and a zero\% 5-year disease-free survival [7]. Similarly radiation therapy alone for these patients resulted in poor 3-year survival $(10 \%-25 \%)$ with significant risk for disease recurrence and death, as well as the complications of soft tissue, ribs, heart and lung injury, also brachial plexopathy, lymphedema, chest wall fibrosis, skin ulceration, and skin necrosis [7]. Although the combination of surgery and radiotherapy decreased incidence of local failure, a high frequency of distant metastasis was seen after either treatment approaches. The introduction of multimodality treatment with the addition of chemotherapy has resulted in improvement of disease-free survival particularly in stage IIIA [4] [7].

Neoadjuvant chemotherapy (NCT) was developed in 1970 and is used before local treatment in LABC to downstage the primary tumor to make subsequent surgery easier, hoping to eliminate distant occult metastasis to prolong survival [8]. The natural history of this disease has been changed dramatically by the introduction of these combined modality therapies with the 5 -year survival rate of 35\% - 60\% commonly are reported [5]. Despite the theoretical and experimental data indicating the survival superiority of neoadjuvant chemotherapy over postoperative adjuvant chemotherapy, the role of neoadjuvant in staging remains unclear [8] [9], and there are few studies that compare this approach to 
postoperative treatment [4] [10]. The purpose of this study is to examine the disease-free survival (DFS) and overall survival (OAS) of patients with LABC treated with NCT, surgery, and radiotherapy to patients treated by primary surgery followed by adjuvant chemotherapy and radiotherapy and to study the prognostic impact of menopausal status and extent of surgical resection on these survival parameters.

\section{Material and Methods}

Eligible patients for this study are those with technically resectable, non-inflammatory locally advanced cancer breast (stage III A \& B) all other patients diagnosed with breast cancer were excluded. 112 patients with previous criteria were treated at King Abdul Aziz Hospital and Oncology Center-Jeddah-Kingdom of Saudi Arabia between January 2011 and December 2015. These patients were treated with multimodality therapy and had been classified into two groups according to whether they were initially treated by neoadjuvant chemotherapy or by surgery. The first group (NCT) included 42 patients who were primarily treated with neoadjuvant chemotherapy followed by surgery after biopsy proven carcinoma and staging work-up at the oncology center. Those patients received FAC regimen (5 fluorouracil, doxorubicin, and cyclophosphamide), then followed by surgery in the form of modified radical mastectomy or conservative resection and axillary clearance. All patients received postoperative radiotherapy to chest wall or breast and lymphatic drainage site. Adjuvant hormone therapy (antiestrogen) was given to all patients regardless of the hormone receptor status plus adjuvant four courses of chemotherapy (postoperatively). The second group (primary surgery) included 70 patients who were referred to the oncology center for postoperative adjuvant management. Those patients have been staged and defined as locally advanced cancer breast (IIIA \& IIIB) by surgical and pathology teams. Patients were operated either by modified radical mastectomy or by conservative surgery and axillary clearance. Those patients have been staged and defined as locally advanced cancer breast (IIIA \& IIIB) by surgical and pathology teams. Patients were operated either by modified radical mastectomy or by conservative surgery and axillary clearance. For those patients staging workup was performed, including full blood picture, biochemical profile, chest X-ray (CXR), abdominal ultrasonography, bone scan and study of left ventricular ejection fraction by MUGA scan (the later performed for patients who were planned to receive anthracycline).

These second group patients were treated with triple modality, i.e., surgery followed by adjuvant chemotherapy ( 6 courses of CMF or FAC) followed by postoperative radiotherapy to chest wall or breast and peripheral lymphatic. All patients received hormone therapy (antiestrogen) regardless of the hormone receptor status.

Main outcome measures: All the patients have been followed up regularly for 
either recurrence, disease-free interval or death; this continued for two years minimum and five years maximum (median 33 months) checkup in the form of CXR, abdominal ultrasound six monthly and bone scan yearly or whenever clinically indicated. Comparative analysis of the two groups regarding disease-free survival (DFS) and overall survival (OAS) was done. The incidence of disease progression, as well as the type of progression, was studied for all patients and for both groups separately with statistical evaluation of the results. The prognostic significance of extent of surgery and menopausal status were studied and correlated to DFS, OAS and disease progression with statistical evaluation of the results in both groups.

The chemotherapy: Consist of the FAC regimen $\left(5-\mathrm{Fu} 500 \mathrm{mg} / \mathrm{m}^{2} / \mathrm{D} 1\right.$ cyclophosphamide $500 \mathrm{mg} / \mathrm{m}^{2} / \mathrm{D} 1$ doxorubicin $50 \mathrm{mg} / \mathrm{m}^{2} / \mathrm{D} 1$. This course was repeated every three weeks (patients with ejection fraction less than $50 \%$, doxorubicin was replaced by mitoxantrone $12 \mathrm{mg} / \mathrm{m}^{2}$ ). The hormonal treatment was Antiestrogen (Tamoxifen) $20 \mathrm{mg} /$ day orally was prescribed for all patients and continued all over the treatment period and is planned to be taken for five years. The postoperative irradiation used for the treatment of all patients by the linear accelerator to a dose of $5040 \mathrm{cGy} / 28$ fractions for chest wall with an electron beam and peripheral lymphatic's (with photon beam). Patients with whole breast received their treatment with the above-mentioned dose with photon beam, and the primary site was boosted to 6000 - 6500 cGy total dose with an electron beam.

Statistical evaluation: Fisher's exact test was used for comparative statistical significance.

\section{Results}

The median age for the whole group was 46.5 years, and the mean age was 46.19 \pm 14.69 years (range $23-75$ ), and the peak age was in the third and fourth decade, which represents $50 \%$ of all patients (Table 1). No difference was noticed in the median age between neoadjuvant chemotherapy group (NCT) and surgery or adjuvant group (surgery) 48 and 46 years respectively (Table 2)

Premenopausal patients constitute $62.5 \%$ of whole patients while $37.5 \%$ were postmenopausal. In both studied groups (NCT) and (surgery) 57\% and 56\% were premenopausal while $43 \%$ and $35 \%$ were postmenopausal respectively (Table 2). Analysis of survival parameters revealed that the median disease-free survival (DFS) for all patients was 15 months and the median overall survival (OAS) was 24 months and there was no difference in (DFC) in (NCT followed by surgery) group and the primary surgery group (16 months and 15 months) as well as the OAS (22 months and 24 months in NCT and the primary surgery group respectively) (Table 3 and Table 4). The influence of menopausal status revealed a highly significant improved difference in DFS and OAS for postmenopause versus premenopause for all patients with DFS 12 months for menopause versus 24 menopause $(\mathrm{p}=0.01)$. Similarly, OAS was 18 months in 
premenopause versus 27 months for postmenopause $(\mathrm{p}=0.01)$ (Table 3$)$.

These differences were also noted in NCT followed by surgery group where DFS was 10.5 months for premenopause and 29 months for postmenopause with $(\mathrm{p}=0.05)$ and OAS was 15.5 months and 35 months for pre and post menopause respectively $(\mathrm{p}=0.03)$. In the primary surgery group this difference in DFS and OAS for pre and post menopause was statistically insignificant $(\mathrm{p}=0.4)$ (Table 4).

Although the noticed improvement of DFS in NCT followed by surgery group versus the primary surgery group in postmenopausal patients (median 29 months versus 19.5 months), the difference was statistically insignificant ( $p=$ 0.06). Similarly, the OAS was 35 months in the NCT group versus 24 months in the primary surgery group in postmenopausal patients, and also the difference was statistically insignificant ( $\mathrm{p}=0.5$ ) (Table 4). Relating the survival data to the extent of surgery performed revealed that the median DFS was 12 months for patients performed modified radical mastectomy (MRM) and 20 months for those patients performed conservative surgery, but the difference was statistically insignificant $(\mathrm{p}=0.03)$. Similarly the OAS was 24 and 29 months for those performed MRM and conservative surgery respectively with statistically insignificant difference between both groups $(\mathrm{p}=0.06)$. However, when survival parameters and extent of surgery were studied within each treatment group, it revealed a statistically insignificant difference in the surgery group for DFS ( $\mathrm{p}=$ 0.8 ) and OAS ( $\mathrm{p}=0.7)$ for those performing MRM and conservative surgery (Table 5). But in the NCT followed by surgery group, there was statistically a significant difference for DFS $(\mathrm{p}=0.02)$ and OAS $(\mathrm{p}=0.02)$ in favor of patients performing conservative surgery. It was also noted in patients performed MRM that there was a statistically significant difference in favor of the primary surgery group versus NCT followed by surgery group regarding DFS (15 vs. 8 months, p $=0.02$ ) and OAS (24 vs. 15.5 months, $\mathrm{p}=0.02$ ) (Table 5). The effect of primary chemotherapy in (NCT followed by surgery) group revealed that six patients (14.3\%) showed complete clinical remission, 14 patients (33.3\%) showed more than $50 \%$ regression of the primary tumor while 22 patients $(52.4 \%)$ showed less than $50 \%$ regression of the primary tumor. There was no complete pathological remission among patients who achieved complete clinical remission. However, the pathologically free margin was achieved for all patients performed post-chemotherapy conservative excision.

Regarding treatment results, the incidence of metastatic disease was $55.4 \%$ for all patients, $47.6 \%$ in NCT followed by surgery group and $60 \%$ in the primary surgery group with a statistically marginal significant difference between both groups $(\mathrm{p}=0.07)$. Freedom of disease was achieved in $33.9 \%$ of all patients, $28.6 \%$ for NCT followed by surgery group and $37.1 \%$ for the primary surgery group with the statistically marginal significant difference between both groups $(\mathrm{p}=0.08)$. As for local recurrence, only $2 / 70$ of patients in the primary-surgery group (2.9\%) experienced local recurrence versus $10 / 42$ patients $(23.8 \%)$ in NCT 
followed by surgery group. Statistical analysis could not be estimated for this category due to the presence of only two patients in the primary-surgery group (Table 6).

Table 1. Age group distribution in locally advanced cancer breast.

\begin{tabular}{cccc}
\hline & Age groups & No. & $\%$ \\
\hline 1 & $20-30$ & 12 & 10.7 \\
2 & $30-40$ & 28 & 25 \\
3 & $40-50$ & 30 & 26.8 \\
4 & $50-60$ & 14 & 12.5 \\
5 & $60-70$ & 14 & 12.5 \\
6 & $>70$ & 16 & 14.3 \\
Total & & 112 & 100
\end{tabular}

Table 2. Patients characteristic in both groups.

\begin{tabular}{ccc}
\hline Patients characteristic & $\begin{array}{c}\text { Neoadjuvant } \\
\text { followed by } \\
\text { surgery }\end{array}$ & Primary surgery \\
\hline Number & 42 & 70 \\
Age (median) & 48 & 46 \\
Menopause: & 24 & 46 \\
Premenop & 18 & 70 \\
Postmenop & & 12 \\
Tumour status: & & 34 \\
T2 & 30 & 20 \\
T3 & 12 & 4 \\
T4 & & \\
Tx & & 38 \\
Nodal status: & 36 & 24 \\
N1 & 6 & 8 \\
N2 & & 62 \\
Nx & 20 & 8 \\
Surgical procedures: & 22 & \\
Mod. Rad. Mas. & & \\
Conservative surgery & & \\
& & \\
\hline
\end{tabular}

Table 3. Survival parameters in correlation with menopausal status.

\begin{tabular}{cccc}
\hline & No. & $\begin{array}{c}\text { Dis. Free survival } \\
\text { (median) Months }\end{array}$ & $\begin{array}{c}\text { Overall survival } \\
\text { (median) Months }\end{array}$ \\
\hline Premenopause & 70 & $12^{\star}$ & $18^{\star *}$ \\
Postmenopause & 42 & $24^{\star}$ & $27^{\star *}$ \\
Total & 112 & 15 & 24 \\
\hline
\end{tabular}

${ }^{*} \mathrm{p}=0.01,{ }^{*} \mathrm{p}=0.01$ 
Table 4. survival parameters (median values) in correlation to menopausal status in both studied groups.

\begin{tabular}{ccccccc}
\hline & \multicolumn{3}{c}{ Neoadjuvant group } & \multicolumn{3}{c}{ Surgery group } \\
\cline { 2 - 7 } & No. & DFS & OAS & No & DFS & OAS \\
\hline Premenopausal & 24 & $10.5^{*}$ & $15.5^{\mathrm{a}}$ & 46 & $12^{1}$ & $20^{2}$ \\
Postmenopausal & 18 & ${ }^{\mathrm{b}} 29^{*}$ & $35^{\mathrm{a}}$ & 24 & ${ }^{\mathrm{b}} 19.5^{1}$ & $24^{2}$ \\
Total & & 16 & 22 & 35 & 15 & 24 \\
\hline
\end{tabular}

${ }^{*} \mathrm{p}=0.05,{ }^{2} \mathrm{p}=0.4,{ }^{\mathrm{a}} \mathrm{p}=0.03,{ }^{\mathrm{b}} \mathrm{p}=0.6,{ }^{1} \mathrm{p}=0.4$.

Table 5. Survival parameters (median values) in correlation to the extent of surgery in the studied groups.

\begin{tabular}{cccccccccccc}
\hline & \multicolumn{3}{c}{ All patients } & \multicolumn{4}{c}{$\begin{array}{c}\text { Neoadjuvant followed } \\
\text { by surgery group }\end{array}$} & \multicolumn{2}{c}{$\begin{array}{c}\text { Primary surgery } \\
\text { group }\end{array}$} \\
\cline { 2 - 10 } & No. & DFS & OAS & No. & DFS & OAS & No. & DFS & OAS \\
\hline $\begin{array}{c}\text { Modified radical } \\
\text { mastectomy }\end{array}$ & 82 & 12 & 24 & 20 & ${ }^{1} 8_{\mathrm{a}}$ & ${ }^{\mathrm{b}} 15.5^{2}$ & 62 & ${ }^{3} 15^{\mathrm{a}}$ & ${ }^{\mathrm{b}} 24^{+}$ \\
$\begin{array}{c}\text { Conservative surgery }+ \\
\text { axillary clearance }\end{array}$ & 30 & 20 & 29 & 22 & ${ }^{1} 16$ & $35^{2}$ & 8 & ${ }^{3} 15$ & $19^{+}$ \\
$\begin{array}{c}\text { Total } \\
\text { Total }\end{array}$ & 56 & 15 & 24 & 21 & 16 & 22 & 35 & 15 & 24 \\
\hline
\end{tabular}

${ }^{\mathrm{a}} \mathrm{p}=0.02,{ }^{\mathrm{b}} \mathrm{p}=0.02,{ }^{1} \mathrm{p}=0.02,{ }^{3} \mathrm{p}=0.8,{ }^{+} \mathrm{p}=0.7$.

Table 6. Results of treatment in correlation to the treatment strategy adopted for locally advanced cancer breast.

\begin{tabular}{ccccccc}
\hline \multirow{2}{*}{ Patient status } & \multicolumn{2}{c}{ All patients } & \multicolumn{2}{c}{ Neoadjuvant group } & \multicolumn{2}{c}{ Surgery group } \\
\cline { 2 - 7 } & No. & $\%$ & No. & $\%$ & No. & $\%$ \\
\hline Free & 38 & 33.9 & 12 & $28.6^{*}$ & 26 & $37.1^{*}$ \\
& - & - & - & - & - & - \\
Local recurrence & 12 & 10.7 & 10 & 23.8 & 2 & 2.9 \\
Metast. Dis. & 62 & 55.4 & 20 & $47.6^{* *}$ & 42 & $60^{* *}$ \\
Total & 56 & 100 & 21 & 100 & 35 & 100 \\
\hline
\end{tabular}

${ }^{*} \mathrm{p}=0.08,{ }^{* *} \mathrm{p}=0.07$.

\section{Discussion}

Locally advanced breast carcinoma is associated with a poor prognosis; with single treatment modality, i.e., surgery, and/or radiotherapy, results have been consistently dismal [11]. The appropriate management of locally advanced breast cancer is controversial, the trends towards a more effective means of improving response rates and survival have shifted to earlier aggressive treatment, and the strength in the management of LABC lies in the team approach to multimodality care [12]. The sequence of treatment in those patients still has to be optimized since despite the theoretical and experimental data indicating the survival superiority of neoadjuvant followed by surgery over 
primary surgery with adjuvant chemotherapy. The role of neoadjuvant chemotherapy in the treatment of breast cancer remains unclear although downstaging of the primary tumor is confirmed [9]. In the present study which aimed at comparison of patients treated with neoadjuvant chemotherapy followed by surgery and those operated by a primary operation followed by adjuvant chemotherapy, analysis of data revealed that the median age was 46.5 years and the mean was $47.19 \pm 14.69$ years. This median age was also reported from a similar study performed in KFSH-Riyadh study [13]. This mean age is not different from the overall age incidence for breast cancer in Saudi Arabia, which reported mean age at diagnosis to be 48.3 years, Cancer Incidence Saudi Arabia [14]. Premenopausal patients comprise $62.5 \%$ of all patients and $37.5 \%$ postmenopausal, and this incidence was similar to that reported by KFSH-Riyadh [15].

Analysis of survival data revealed that the median DFS in our patients was 16 months, a similar figure (17 months) was reported in a similar study [15] [16]. No difference in DFS and OAS was noticed in patients treated by NCT followed by surgery or by primary surgery, and this finding has been documented by [17] who compared preoperative with postoperative therapy in operable breast cancer patients including LABC cases and found no difference in DFS and OAS between pre and postoperative chemotherapy. Cunningham et al. and Kuerer et al. also found such significant difference in DFS and OAS between NCT and postoperative adjuvant treatment [10] [16].

This significance of menopausal status in this study showed that DFS and OAS were significantly better in the older age group (postmenopause). This finding has been studied by Crowe et al. who found that younger patients had more estrogen receptor negative tumors and a greater number of positive lymph nodes, and he concluded that younger patients as a group have more aggressive and advanced cancer compared to older patients, but it should not be used alone for management decision [17]. This was also reported by Newman et al. who confirmed that younger women tend to present with more locally advanced breast cancer and their tumors may have a different response to treatment, compared to older patients and hence this significant difference in survival is a reflection of the aggressive nature of the disease in younger age group [18] [19].

The second finding concerning age in our results was noticed by inspecting the insignificant difference in DFS and OAS for pre and postmenopausal patients in the primary surgery group and also postmenopausal patients in either group. This finding could suggest the possibility that the younger age group might benefit from earlier surgical interference in premenopausal patients especially those who initially showed minimal response to chemotherapy (after two courses). Although, the DFS and OAS were more or less similar in both groups; however previous surgery for premenopausal patients may abolish this significant difference in survival between pre and post menopause for patients treated with neoadjuvant chemotherapy. 
Considering the extent of the surgery, the insignificant difference in survival parameters (DFS and OAS) for all patients and also for patients in primary surgery group could be explained by the fact that the main problem in patients with $\mathrm{LABC}$ is the distant failure regardless the locoregional control of the disease [19] [20]. For this reason, recent trials and utilizing aggressive chemotherapy with newer agents like Texas [21] [22] or by increasing dose intensity in conjunction with growth factors to increase the response of the tumors to primary chemotherapy which may improve the survival and this is reported in southwest oncology group phase II trials [23].

On the other hand in NCT followed by surgery group, there was the discrepancy in the results since there was the significant difference in survival parameters (DFS and OAS) favoring patients who performed conservative surgery. However, this finding could be explained by the fact that patients who performed conservative surgery were those who showed excellent response to primary chemotherapy and they achieved better results than those who showed minimal response to chemotherapy and consequently performed modified radical mastectomy did. These results were also reported by Schwartz et al. who showed in their series of patients treated by neoadjuvant chemotherapy a five year disease-free survival $56 \%$ for those having mastectomy and $77 \%$ for those having breast conservation and five years overall survival was $67 \%$ for those performed mastectomy and $80 \%$ for those having breast conservation [24]. Recent trials currently are using aggressive chemotherapy to achieve a higher remission clinically and pathologically to improve DFS and OAS [25] [26] [27]. Worth mentioning that those responsive patients to primary chemotherapy who underwent conservative surgery might be sensitive also to the adjuvant chemotherapy postoperatively and that is why they showed improved survival parameters in comparison with all other subsets of patients.

Regarding treatment results, freedom of disease was noticed in $37.1 \%$ of primary surgery group vs. $28.6 \%$ in NCT followed by surgery group with the borderline significant $(\mathrm{p}=0.08)$. This could be explained by the presence of only one patient with local recurrence in the primary surgery group vs. 5 in the NCT followed by surgery group, and this is the consequence of a large number of patients performed conservative surgery in the NCT group (22 patients) versus only eight patients in the primary surgery group. This finding was reported by Caudle et al., who found that mastectomy substantially decreased locoregional recurrence, but distant metastases were a significant component of failure [28]. This was noticed in this work, where there is $60 \%$ incidence of metastatic disease in the primary surgery group vs. $47.6 \%$ in NCT followed by surgery group with the borderline significant difference $(\mathrm{p}=0.07)$. This could be explained by the fact that most LABC patients would be already harboring micrometastases at their initial diagnosis, that should be primarily treated by chemotherapy as it was suggested that chemotherapy might modulate the host environment to prevent tumor cell migration [29] [30] [31]. 


\section{Conclusion}

The results of this study showed that primary surgery followed by postoperative therapy is comparable to neoadjuvant treatment followed by surgery in LABC regarding disease-free survival, overall survival, distant failure, and disease control. However, local recurrence was higher in NCT followed by surgery group because of the favorability of conservative resection in both patients and surgeons side once they got tumor shrinkage, but we have to give attention to what was reported about this situation by [26] who found that chemotherapy is useful in reducing tumor size to allow surgical resection but does not sterilize the breast of cancer, and they caution against the use of any surgery less than total mastectomy in partially responsive tumor if optimal local control is to be achieved in locally advanced breast cancer. Secondly, the post menopause (older age) patients felt much better than the younger generation regarding survival parameters particularly with NCT followed by surgery group. So, we recommend that younger patients who deserve neoadjuvant chemotherapy have to be treated aggressively by surgery not less than mastectomy. The other alternative is the use of a newer chemotherapeutic agent or increasing dose intensity of chemotherapy to obtain a higher clinical and pathological remission so that conservative surgery could be performed with optimal local control and reducing distant failure [29] [30]. Neoadjuvant chemotherapy will also provide a useful biological model to assess the effects of systemic treatment on the primary tumor and regional metastases, in addition to hoping to reduce distant failure.

\section{Conflicts of Interest}

The author declares no conflict of interest.

\section{References}

[1] Singletary, S.E., Allred, C., Ashley, P., et al. (2002) Revision of the American Joint Committee on Cancer Staging System for Breast Cancer. Journal of Clinical Oncology, 20, 3628-3636. https://doi.org/10.1200/JCO.2002.02.026

[2] Simos, D., Clemons, M., Ginsburg, O.M. and Jacobs, C. (2014) Definition and Consequences of Locally Advanced Breast Cancer. Current Opinion in Supportive and Palliative Care, 8, 33-38

[3] Cristofanilli, M., Buzdar, A.U. and Hortobágyi, G.N. (2003) Update on the Management of Inflammatory Breast Cancer. The Oncologist, 8, 141-148.

https://doi.org/10.1634/theoncologist.8-2-141

[4] Caudle, A.S. and Kuerer, H.M. (2014) Breast Conservation Therapy after Neoadjuvant Chemotherapy: Optimization of a Multimodality Approach. Journal of Surgical Oncology, 110, 32-36. https://doi.org/10.1002/jso.23595

[5] Yalcin, B. (2013) Overview on Locally Advanced Breast Cancer: Defining, Epidemiology, and Overview on Neoadjuvant Therapy. Experimental Oncology, 35, 250-252.

[6] Guilherme, F., Angotti, C., Cristovam, S., Lucas Faria, A., Maria, M., João, S., et al. (2017) Breast-Conserving Surgery in Locally Advanced Breast Cancer Submitted to Neoadjuvant Chemotherapy. Safety and Effectiveness Based on Ipsilateral Breast 
Tumor Recurrence and Long-Term Follow-Up. Clinics (Sao Paulo), 72, 134-142. https://doi.org/10.6061/clinics/2017(03)02

[7] Ellis, G.K., Barlow, W.E., Gralow, J.R., Hortobagyi, G.N., Russell, C.A., Royce, M.E., et al. (2011) Phase III Comparison of Standard Doxorubicin and Cyclophosphamide versus Weekly Doxorubicin and Daily Oral Cyclophosphamide plus Granulocyte Colony-Stimulating Factor as Neoadjuvant Therapy for Inflammatory and Locally Advanced Breast Cancer: SWOG 0012. Journal of Clinical Oncology, 29, 1014-1021. https://doi.org/10.1200/JCO.2009.27.6543

[8] Mauri, D., Pavlidis, N. and Ioannidis, J.P. (2005) Neoadjuvant versus Adjuvant Systemic Treatment in Breast Cancer: A Meta-Analysis. Journal of the National Cancer Institute, 97, 188-194. https://doi.org/10.1093/jnci/dji021

[9] Wang, H.C. and Lo, S.S. (1996) Future Prospects of Neoadjuvant Chemotherapy in the Treatment of Primary Breast Cancer. Seminars in Surgical Oncology, 12, 59-66. https://doi.org/10.1002/(SICI)1098-2388(199601/02)12:1<59::AID-SSU9>3.0.CO;2-5

[10] Cuningham, J.D., Weiss, S.E., Ahmad, S., Bratton, J.H., Bleiweiss, I.J., Tarter, P.I. and Brower, S.T. (1998) The Efficacy of Neoadjuvant Chemotherapy Compared to Postoperative Therapy in the Treatment of Locally Advanced Breast Cancer. Cancer Investigation, 16, 86-96. https://doi.org/10.3109/07357909809039761

[11] Karlesson, Y.A., Melstrom, P.O., Hatschek, T., Fornander, T.G., Soderberg, M., Bengtsson, Y.A., et al. (1998) Multimodality Treatment of 128 Patients with Locally Advanced Breast Carcinoma in the Era of Mammography Screening Using Standard Polychemotherapy with 5-FU Epirubicin and Cyclophosphamide, Prognostic and Therapeutic Implications. Cancer, 83, 936-947.

[12] Singletary, S.E., Hortobagyi, G.N. and Kroll, S.S. (1995) Surgical and Medical Management of Local-Regional Treatment Failure in Advanced Primary Breast Cancer. Surgical Oncology Clinics of North America, 4, 671-684.

[13] Ezzat, A.A., Ibrahim, E.M., Raja, M.A., Al-Sobhi, S., Rostom, A. and Stuart, R.K. (1999) Locally Advanced Breast Cancer in Saudi Arabia: High Frequency of Stage III in a Young Population. Medical Oncology, 16, 95-103. https://doi.org/10.1007/BF02785842

[14] (2016) Cancer Incidence SA June 2016. Cancer Incidence Report, Saudi Arabia (2013-2015), Kingdom of Saudi Arabia, Ministry of Health, National Cancer Registry.

[15] Lee, M.C. and Newman, L.A. (2007) Management of Patients with Locally Advanced Breast Cancer. Surgical Clinics of North America, 87, 379-398. https://doi.org/10.1016/j.suc.2007.01.012

[16] Fisher, B., Bryant, J., Wol, A.N., Mamounas, E., Brown, A., Fisher, E.R., et al. (1998) Effect of Postoperative Chemotherapy on the Outcome of Women with Operable Breast Cancer. Journal of Clinical Oncology, 16, 2672-2685.

https://doi.org/10.1200/JCO.1998.16.8.2672

[17] Kuerer, H.M., Newman, L.A., Smith, T.L., Ames, F.C., Hunt, K.K., Buda, A.U., Hortobagyi, G.N. and Singletary, S.E. (1999) Clinical Course of Breast Cancer Patients with Complete Pathologic Primary Tumor and Axillary Lymph Nodes Response to Doxorubicin-Based Neoadjuvant Chemotherapy. Journal of Clinical Oncology, 17, 468-496. https://doi.org/10.1200/JCO.1999.17.2.460

[18] Crow, J.P., Gordon, N.H., Shank, R.R., Zollinger, R.M., Brumberg, D.J. and Shuck, J.M. (1994) Age Does Not Predict Cancer Outcome. The Archives of Surgery, 129, 483-496. https://doi.org/10.1001/archsurg.1994.01420290029004

[19] Newman, L.A., Kuerer, H.M., Singh, G., Bucchloz, T.A., Hunt, K.K., Ames, F.C. and 
Singletary, S.E. (1998) Age-Related Difference in the Utilization and Impact of Multimodality Therapy on the Survival of Black Breast Cancer Patients. 34th Annual Meeting on Program/Proceedings of the American Society of Clinical Oncology, Vol. 17, May 1998.

[20] Harris, J., Morrow, M. and Norton, L. (1997) Malignant Tumors of the Breast. In: DeVita, V.T., Hellman, S. and Rosenberg, S.A., Eds., Cancer Principles and Practice of Oncology, 5th Edition, Lippincott-Raven Publishers, Hagerstown, Ch. 36, Sec. 2.

[21] Philiph, P.A., Penick, N.L., Vashanpey An, U., Simon, M.S., Lorusso, P., Chaplan, R., et al. (2000) Phase II Study of Doxorubicin, Docetaxel and 5 Fu (FAT) in Patients with Locally Advanced Breast Cancer. 36th Annual Meeting on Program/Proceedings of the American Society of Clinical Oncology, Vol. 19, May 2000.

[22] Esteva, F.J., Rosoles, M.F., Esparazaguerre, L.T., Sahin, A.A., Soss, M.I., Booser, D.J., et al. (2000) Phase II Trial of Primary Chemotherapy with Docetaxel and Doxorubicin in Locally Advanced Breast Cancer-Clinical and Pathological Results. 36th Annual Meeting on Program/Proceedings of the American Society of Clinical Oncology, Vol. 19, May 2000.

[23] Ellis, G.K., Green, S.J., Livingstone, G.T., Budd, S.E., Rivkin, J.K., Gandura, G.R. and Martino, S. (2000) Neoadjuvant Doxorubicin, Cyclophosphamide and G-CSF $(\mathrm{AC}+\mathrm{G})$ for Locally Advanced Breast Cancer (LABC) Southwest Oncology Phase II Trial. 36th Annual Meeting on Program/Proceedings of the American Society of Clinical Oncology, Vol. 19, May 2000.

[24] Schwartz, G.F., Birchansky, C.A., Komamicky, L.T., Mansfield, C.M., Cantor, R.I., et al. (1994) Induction Chemotherapy Followed by Breast Conservation for Locally Advanced Carcinoma of the Breast. Cancer, 73, 362-369.

https://doi.org/10.1002/1097-0142(19940115)73:2<362::AID-CNCR2820730221>3.0 .CO;2-L

[25] Murthy, S.M., Reid, S.E., Jonarden, S., Scenlon, C.F. and Khankar, J.D. (1999) Prevention of Breast Cancer Relapse by Neoadjuvant Chemotherapy-Status of the Mechanism. 36th Annual Meeting on Program/Proceedings of the American Society of Clinical Oncology, Vol. 18, May 1999.

[26] Kent, A.L., Eaton, M., Marshal, N. and Humeniuk, V. (1995) Locally Advanced Breast Cancer Is Surgery Warranted Following, Chemotherapy. ANZ Journal of Surgery, 65, 229-332. https://doi.org/10.1111/j.1445-2197.1995.tb00617.x

[27] Green, M.C., Buzdar, A.U., Smith, T., et al. (2005) Weekly Paclitaxel Improves Pathologic Complete Remission in Operable Breast Cancer When Compared with Paclitaxel Once Every 3 Weeks. Journal of Clinical Oncology, 23, 5983-5992. https://doi.org/10.1200/JCO.2005.06.232

[28] Taylor, M.E., Taylor, M.E., Perez, C.A. and Levitt, S.H. (1997) Breast: Locally Advanced (T3 and T4), Inflammatory and Recurrent Tumors. In: Perez, C.A. and Brady, L.W., Eds., Principles and Practice of Radiation Oncology, 3rd Edition, Lippincott-Raven Publishers, Hagerstown, Ch. 51.

[29] Ellis, G.K., Green, S.J., Russell, C.A., et al. (2006) SWOG 0012, a Randomized Phase III Comparison of Standard Doxorubicin and Cyclophosphamide Followed by Weekly Paclitaxel versus Weekly Doxorubicin and Daily Oral Cyclophosphamide plus G-CSF Followed by Weekly Paclitaxel as Neoadjuvant Therapy for Inflammatory and Locally Advanced Breast Cancer. Journal of Clinical Oncology, 24, LBA537.

[30] Von Minckwitz, G., Raab, G., Caputo, A., et al. (2005) Doxorubicin with Cyclophosphamide Followed by Docetaxel Every 21 Days Compared with 
Doxorubicin and Docetaxel Every 14 Days as Preoperative Treatment in Operable Breast Cancer: The GEPARDUO Study of the German Breast Group. Journal of Clinical Oncology, 23, 2676-2685. https://doi.org/10.1200/JCO.2005.05.078

[31] Papademetriou, K., Ardavanis, A. and Kountourakis, P. (2010) Neoadjuvant Therapy for Locally Advanced Breast Cancer: Focus on Chemotherapy and Biological targeted Treatments' Armamentarium. Journal of Thoracic Disease, 2, 160-170. 